\title{
Unveiling Order behind Complexity: Coexistence of Ferromagnetism and Bose-Einstein Condensation
}

\author{
C.D. Batista, G. Ortiz, and J.E. Gubernatis \\ Theoretical Division, Los Alamos National Laboratory, Los Alamos, NM 87545
}

(Received November 1, 2018)

\begin{abstract}
We present an algebraic framework for identifying the order parameter and the possible phases of quantum systems that is based on identifying the local dimension $N$ of the quantum operators and using the $S U(N)$ group representing the generators of generalized spin-particle mappings. We illustrate this for $N=3$ by presenting for any spatial dimension the exact solution of the bilinearbiquadratic $S=1$ quantum Heisenberg model at a high symmetry point. Through this solution we rigorously show that itinerant ferromagnetism and Bose-Einstein condensation may coexist.
\end{abstract}

Introduction. One of the fascinating things about matter is the different phases it exhibits. One way or another, identifying these phases and the transitions between them occupies the interests and activities of most condensed-matter physicists, physical chemists, and field theorists. What is typically used as a working principle is Landau's postulate of an order parameter (OP). While one generally knows what to do if the OP is known, Landau's postulate gives no procedure for finding it. This problem is particularly complex if the symmetries of the system are hidden and "veil" the nature of the order. For example, it took a while to recognize that the staggered magnetization was the OP for anti-ferromagnetism.

In this Letter, we present a simple algebraic framework for identifying OPs and the possible zero temperature phases (ground states (GSs)) of quantum systems of spins, fermions, and bosons. Central to this framework is identifying the local dimension $N$ of the quantum operators, which sets the order of the largest Lie group that can be used to describe those systems. The OP is constructed from this hierarchical group, and the embedded subgroups are identified to establish a hierarchical classification of the possible broken symmetry phases.

To place this framework in more familiar terms, we recall the Jordan-Wigner [1] and Matsubara-Matsuda [2] spin-particle transformations that map $S=\frac{1}{2}$ spins into spinless fermions and into hard-core (HC) bosons. In the latter case, this mapping demonstrated that ferromagnetism in a localized spin model was isomorphic to BoseEinstein (BE) condensation in a $\mathrm{HC}$ boson model. We note that these two venerable transformations are special cases of the generalized transformations we recently introduced [3, [1] and in fact they should be viewed as the same transformation: Their local dimension is $N=2$, corresponding to the up and down states of a spin or the occupied and unoccupied states of a spinless fermion or a HC boson. The hierarchical group is $S U(2)$, and the broken symmetry states break either this symmetry in the ferromagnetic (FM) case or the $U(1)$ symmetry of its subgroup in the BE condensation case.

The key to the general framework we are proposing is the existence of a general set of $S U(N)$ spin-particle transformations [3,5], fundamental for devising a general theoretical scheme to understand the order hidden in complex behavior. More simply put, through the generalized spin-particle transformations, features that are subtle and hard to identify in one representation (hidden symmetries) can become prominent and easy to analyze in another (explicit symmetries). Indeed, these mappings connect seemingly unrelated physical phenomena establishing equivalence relations among them. In the hierarchical group, all elements of the operator basis are symmetry generators $[6]$. This allows one to study the coexistence and competition of phases, like magnetism and superconductivity, with the corresponding OPs derived from the subgroup generators embedded in the largest global symmetry group of the problem.

The $N=2$ case is too simple to illustrate all these points. We can, however, illustrate them by moving up to $N=3$ and be very concrete by studying a $S=1$ bilinear-biquadratic quantum spin Hamiltonian. In fact, we will present the exact solution to this problem at a point of high symmetry in any spatial dimension. Using our generalized spin-particle transformations, we show this model happens to map onto a lattice gas model of interacting particles with an internal quantum number (two-flavored HC bosons). The exact solution transparently exhibits two simultaneously broken continuous symmetries associated to the formation of a polarized BE condensate. In this way, we rigorously show that these two quantum orderings can coexist [7]. This novel result emerges naturally by identifying the underlying highest rank algebra $(s u(3))$, providing the framework to determine the possible broken symmetries and understand the role of symmetry-reducing perturbations. Using it, we will construct the complex phase diagram in a neighborhood of the model's high symmetry point.

Ferromagnetism and BE Condensation. The Hamiltonian we studied is [8]

$$
H_{\theta}=J \sqrt{2} \sum_{\langle\mathbf{i}, \mathbf{j}\rangle}\left[\cos \theta \mathbf{S}_{\mathbf{i}} \cdot \mathbf{S}_{\mathbf{j}}+\sin \theta\left(\mathbf{S}_{\mathbf{i}} \cdot \mathbf{S}_{\mathbf{j}}\right)^{2}\right]
$$


where $J<0$ and summation is over bonds $\langle\mathbf{i}, \mathbf{j}\rangle$ of a regular $d$-dimensional lattice with $N_{s}$ sites. A spin $S=1$ operator $\mathbf{S}_{\mathbf{i}}$ is associated with lattice site $\mathbf{i}$ and locally satisfies the $s u(2)$ Lie algebra.

The case $H_{1}=H_{\theta=\frac{\pi}{4}}$ can be conveniently written in a $\left(s=\frac{1}{2}\right) \mathrm{HC}$ boson representation $\left(\bar{n}_{\mathbf{j} \sigma} \bar{n}_{\mathbf{j} \sigma^{\prime}}=\delta_{\sigma \sigma^{\prime}} \bar{n}_{\mathbf{j} \sigma}\right)$ [9]

$$
\frac{S_{\mathbf{j}}^{+}}{\sqrt{2}}=\bar{b}_{\mathbf{j} \uparrow}^{\dagger}+\bar{b}_{\mathbf{j} \downarrow}, \frac{S_{\mathbf{j}}^{-}}{\sqrt{2}}=\bar{b}_{\mathbf{j} \uparrow}+\bar{b}_{\mathbf{j} \downarrow}^{\dagger}, S_{\mathbf{j}}^{z}=\bar{n}_{\mathbf{j} \uparrow}-\bar{n}_{\mathbf{j} \downarrow},
$$

as an extended $t-J$ like Hamiltonian,

$$
\begin{aligned}
H_{1} & =J \sum_{\langle\mathbf{i}, \mathbf{j}\rangle, \sigma}\left(\bar{b}_{\mathbf{i} \sigma}^{\dagger} \bar{b}_{\mathbf{j} \sigma}+\text { H.c. }\right)+2 J \sum_{\langle\mathbf{i}, \mathbf{j}\rangle} \mathbf{s}_{\mathbf{i}} \cdot \mathbf{s}_{\mathbf{j}} \\
& +2 J \sum_{\langle\mathbf{i}, \mathbf{j}\rangle}\left(1-\frac{\bar{n}_{\mathbf{i}}+\bar{n}_{\mathbf{j}}}{2}+\frac{3}{4} \bar{n}_{\mathbf{i}} \bar{n}_{\mathbf{j}}\right),
\end{aligned}
$$

with $\mathbf{s}_{\mathbf{j}}=\frac{1}{2} \bar{b}_{\mathbf{j} \mu}^{\dagger} \boldsymbol{\sigma}_{\mu \nu} \bar{b}_{\mathbf{j} \nu}$ a $s=\frac{1}{2}$ operator $(\boldsymbol{\sigma}$ denoting Pauli matrices), and $\bar{n}_{\mathbf{j}}=\bar{b}_{\mathbf{j} \uparrow}^{\dagger} \bar{b}_{\mathbf{j} \uparrow}+\bar{b}_{\mathbf{j} \downarrow}^{\dagger} \bar{b}_{\mathbf{j} \downarrow}$. This last form in turn can be rewritten as

$$
H_{1}=2 J \sum_{\langle\mathbf{i}, \mathbf{j}\rangle} P_{s}(\mathbf{i}, \mathbf{j})
$$

where $P_{s}(\mathbf{i}, \mathbf{j})=P_{s}^{2}(\mathbf{i}, \mathbf{j})$ is the projector onto the symmetric subspace $(S=0,2)$ corresponding to the bond $\langle\mathbf{i}, \mathbf{j}\rangle$ which indicates that if one finds a state that is symmetric under the permutation of nearest neighbors $\mathbf{r}_{\mathbf{i}}$ and $\mathbf{r}_{\mathbf{j}}$, then that state is the GS.

For a system of $\mathcal{N} \mathrm{HC}$ bosons the GS is

$$
\left|\Psi_{0}\left(\mathcal{N}, S_{z}\right)\right\rangle=\left(\tilde{b}_{\mathbf{0} \uparrow}^{\dagger}\right)^{\mathcal{N}_{\uparrow}}\left(\tilde{b}_{\mathbf{0} \downarrow}^{\dagger}\right)^{\mathcal{N}_{\downarrow}}|0\rangle,
$$

$\left(\mathcal{N}=\mathcal{N}_{\uparrow}+\mathcal{N}_{\downarrow} \leq N_{s}\right)$ with an energy $E_{0}=J N_{s} \mathrm{z}(\mathrm{z}$ is the coordination of the lattice) and a total $S_{z}=\frac{\mathcal{N}_{\uparrow}-\mathcal{N}_{\downarrow}}{2}$. The operator $\tilde{b}_{\mathbf{0} \sigma}^{\dagger}$ is the $\mathbf{k}=\mathbf{0}$ component of $\bar{b}_{\mathbf{j} \sigma}^{\dagger}$, i.e., $\tilde{b}_{\mathbf{k} \sigma}^{\dagger}=\frac{1}{\sqrt{N_{s}}} \sum_{\mathbf{j}} \exp \left[i \mathbf{k} \cdot \mathbf{r}_{\mathbf{j}}\right] \bar{b}_{\mathbf{j} \sigma}^{\dagger}$. The quasihole and quasiparticle excited states are

$$
\left\{\begin{aligned}
\left|\Psi_{\mathbf{k}}^{h}\left(\mathcal{N}, S_{z}\right)\right\rangle & =\tilde{b}_{\mathbf{k} \sigma}\left|\Psi_{0}\left(\mathcal{N}, S_{z}\right)\right\rangle & & \text { quasihole } \\
\left|\Psi_{\mathbf{k}}^{p}\left(\mathcal{N}, S_{z}\right)\right\rangle & =\tilde{b}_{\mathbf{k} \sigma}^{\dagger}\left|\Psi_{0}\left(\mathcal{N}, S_{z}\right)\right\rangle & & \text { quasiparticle }
\end{aligned}\right.
$$

with the excitation energy of each being $\omega_{\mathbf{k}}=$ $J \mathrm{z}\left(\frac{1}{\mathrm{z}} \sum_{\nu} e^{i \mathbf{k} \cdot \mathbf{e}_{\nu}}-1\right)$ where the sum runs over the vectors $\mathbf{e}_{\nu}$ which connect a given site to its $\mathbf{z}$ nearest neighbors. In the $|\mathbf{k}| \rightarrow 0$ limit, $\omega_{\mathbf{k}} \rightarrow 0$.

Clearly the GS in Eq. . is a FM BE condensate with any partial spin polarization, and the form of the result is independent of the spatial dimensionality of the lattice. We note that different values of $S_{z}$ correspond to the different orientations of the magnetization $\mathcal{M}$ associated to the broken $S U(2)$ spin rotational symmetry of the GS. We also note that the degeneracy of states with different number of particles $\mathcal{N}$ indicates a broken $U(1)$ charge symmetry (conservation of the number of particles) associated to the BE condensate [10]. A signature of $\mathrm{BE}$ condensation is the existence of off-diagonal long-range order (ODLRO) in the correlation function $\Phi_{\sigma \sigma^{\prime}}(\mathbf{i} \mathbf{j})=\left\langle\bar{b}_{\mathbf{i} \sigma}^{\dagger} \bar{b}_{\mathbf{j} \sigma^{\prime}}\right\rangle$. When $\mathcal{N}_{\uparrow}$ and $\mathcal{N}_{\downarrow}$ are both of order $N_{s}$, there are two eigenvectors with eigenvalues of order $N_{s}$ and the condensate is thus a mixture.

We can easily compute the magnetization $\mathcal{M}$ and phase coherence of these various (non-normalized) degenerate GSs for a given density $\rho=\frac{\mathcal{N}}{N_{s}}$. For example, in the fully polarized case, $\mathcal{N}=\mathcal{N}_{\uparrow}, \mathcal{M}=\left\langle S_{\mathbf{j}}^{z}\right\rangle=\rho$, and the ODLRO $\Phi_{\uparrow \uparrow}(\mathbf{i j})=\frac{\rho(1-\rho)}{1-\epsilon}\left(\mathbf{r}_{\mathbf{i}} \neq \mathbf{r}_{\mathbf{j}}\right)$, where $\epsilon=1 / N_{s}$. Similarly, the two-particle correlation function $\left\langle\Delta_{\mathbf{i}}^{\dagger} \Delta_{\mathbf{j}}\right\rangle=\Phi_{\uparrow \uparrow}(\mathbf{i j}) \frac{(\rho-\epsilon)(1-\rho-\epsilon)}{(1-2 \epsilon)(1-3 \epsilon)}$, where $\Delta_{\mathbf{i}}^{\dagger}=\bar{b}_{\mathbf{i} \uparrow}^{\dagger} \bar{b}_{\mathbf{i}+\boldsymbol{\delta} \uparrow}^{\dagger}$ [11]. Therefore the exact GS has two spontaneously broken continuous symmetries (see Fig. 1).

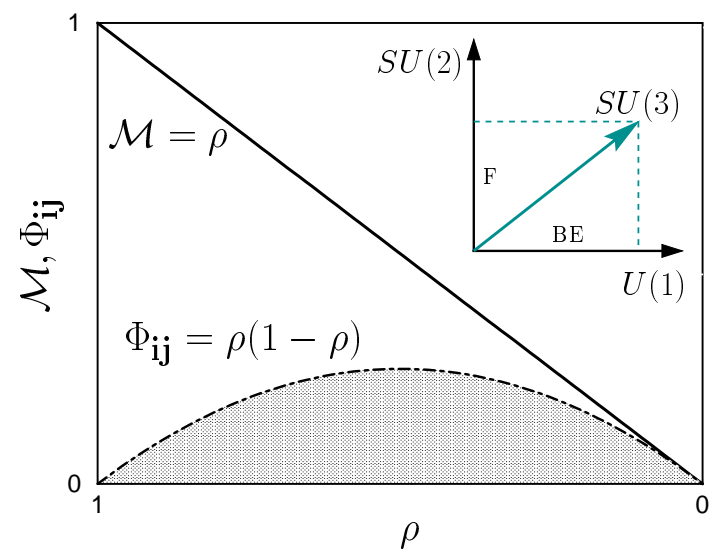

FIG. 1. Coexistence of ferromagnetism and Bose-Einstein condensation. The correlation function $\Phi_{\uparrow \uparrow}(\mathbf{i j})=\Phi_{\mathbf{i j}}$ is expressed in the thermodynamic limit. The inset schematically displays the $S U(3)$ order parameter living in an eight-dimensional space with projections onto the $S U(2)$ (F: ferromagnet) and $U(1)$ (BE: Bose-Einstein condensate) axes.

The exact solution defines the features of the phase diagram that our proposed framework must qualitatively admit. We will see below that both OPs (magnetization and phase), as promised, are embedded in an $S U(3)$ OP. We remark that the phase coexistence in the boson representation maps back to a $S=1 \mathrm{FM}$ phase coexisting with another spin phase. To see this consider the state $\left|\Psi_{0}(\mathcal{N}, 0)\right\rangle$ for which $\left\langle S_{\mathbf{i}}^{z}\right\rangle=\left\langle S_{\mathbf{i}}^{x}\right\rangle=\left\langle S_{\mathbf{i}}^{y}\right\rangle=0$, which implies that it is a singlet state in the $S=1$ representation. We will show below that this other phase has a pure spin-nematic ordering.

What can be said about the fermionic extended $t-J$ Hamiltonian (i.e., Eq. 3 with $\mathrm{HC}$ bosonic operators replaced by "constrained" fermions)? Obviously, the GS energies of both fermionic and bosonic Hamiltonians coincide at $\rho=0,1$. For $0<\rho<1$, one can show that $E_{0}($ fermion $)>E_{0}($ boson $)=J N_{s} z$, indicating that the fermionic system phase segregates [5].

The Framework Illustrated. In Ref. [5], we generalized the spin-particle transformations to spins satisfying the 
$S U(N)$ Lie bracket relations. In particular, the fundamental ("quark") representations of $S U(N)$ are mapped onto an algebra of $\mathrm{HC}$ bosons [5] with $N_{f}=N-1$ flavors

$$
\begin{aligned}
& \mathcal{S}^{\alpha \beta}(\mathbf{j})=\bar{b}_{\mathbf{j} \alpha}^{\dagger} \bar{b}_{\mathbf{j} \beta}-\frac{\delta_{\alpha \beta}}{N} \\
& \mathcal{S}^{\alpha 0}(\mathbf{j})=\bar{b}_{\mathbf{j} \alpha}^{\dagger}, \mathcal{S}^{0 \beta}(\mathbf{j})=\bar{b}_{\mathbf{j} \beta}, \\
& \mathcal{S}^{00}(\mathbf{j})=\frac{N_{f}}{N}-\sum_{\alpha=1}^{N_{f}} \bar{b}_{\mathbf{j} \alpha}^{\dagger} \bar{b}_{\mathbf{j} \alpha}=-\sum_{\alpha=1}^{N_{f}} \mathcal{S}^{\alpha \alpha}(\mathbf{j})
\end{aligned}
$$

where $1 \leq \alpha, \beta \leq N_{f}$ run over the set of bosonic flavors, $\mathbf{j}$ is the site index, and $\bar{b}_{\mathbf{j} \alpha}^{\dagger}=b_{\mathbf{j} \alpha}^{\dagger} \prod_{\beta}\left(1-n_{\mathbf{j} \beta}\right) . S^{\mu \nu}(\mathbf{j})(0 \leq$ $\left.\mu, \nu \leq N_{f}\right)$ are the components of the $S U(N) \operatorname{spin}\left(N^{2}-1\right.$ linearly independent components). These components are generators of an $s u(N)$ Lie algebra with commutation relations $\left[\mathcal{S}^{\mu \mu^{\prime}}(\mathbf{j}), \mathcal{S}^{\nu \nu^{\prime}}(\mathbf{j})\right]=\delta_{\mu^{\prime} \nu} \mathcal{S}^{\mu \nu^{\prime}}(\mathbf{j})-\delta_{\mu \nu^{\prime}} \mathcal{S}^{\nu \mu^{\prime}}(\mathbf{j})$. In particular, for $N=3$ [12]

$$
\mathcal{S}(\mathbf{j})=\left(\begin{array}{ccc}
\frac{2}{3}-\bar{n}_{\mathbf{j}} & \bar{b}_{\mathbf{j} \uparrow} & \bar{b}_{\mathbf{j} \downarrow} \\
\bar{b}_{\mathbf{j} \uparrow}^{\dagger} & \bar{n}_{\mathbf{j} \uparrow}-\frac{1}{3} & \bar{b}_{\mathbf{j} \uparrow}^{\dagger} \bar{b}_{\mathbf{j} \downarrow} \\
\bar{b}_{\mathbf{j} \downarrow}^{\dagger} & \bar{b}_{\mathbf{j} \downarrow}^{\dagger} \bar{b}_{\mathbf{j} \uparrow} & \bar{n}_{\mathbf{j} \downarrow}-\frac{1}{3}
\end{array}\right),
$$

and up to an irrelevant constant we can rewrite $H_{\theta}$ as

$$
\begin{aligned}
H_{\theta} & =J \sqrt{2} \sum_{\langle\mathbf{i}, \mathbf{j}\rangle}\left[\cos \theta \mathcal{S}^{\mu \nu}(\mathbf{i}) \mathcal{S}^{\nu \mu}(\mathbf{j})\right. \\
& \left.+(\sin \theta-\cos \theta) \mathcal{S}^{\mu \nu}(\mathbf{i}) \tilde{\mathcal{S}}^{\nu \mu}(\mathbf{j})\right]
\end{aligned}
$$

where repeated Greek superscripts are summed and

$$
\tilde{\mathcal{S}}(\mathbf{j})=\left(\begin{array}{ccc}
\frac{2}{3}-\bar{n}_{\mathbf{j}} & -\bar{b}_{\mathbf{j} \downarrow}^{\dagger} & -\bar{b}_{\mathbf{j} \uparrow}^{\dagger} \\
-\bar{b}_{\mathbf{j} \downarrow} & \bar{n}_{\mathbf{j} \downarrow}-\frac{1}{3} & \bar{b}_{\mathbf{j} \uparrow}^{\dagger} \bar{b}_{\mathbf{j} \downarrow} \\
-\bar{b}_{\mathbf{j} \uparrow} & \bar{b}_{\mathbf{j} \downarrow}^{\dagger} \bar{b}_{\mathbf{j} \uparrow} & \bar{n}_{\mathbf{j} \uparrow}-\frac{1}{3}
\end{array}\right)
$$

generates the conjugate representation on sublattice $\mathbf{j}$ [14]. This expression for $H_{\theta}$ illustrates the very important result that any nonlinear interaction in the original representation is simply a bilinear term in the new representation when mapped onto the highest rank algebra [0]. In particular, there are certain special points in parameter space where $H_{\theta}$ is highly symmetric. For example, for $\theta=\frac{\pi}{4}$ and $\frac{5 \pi}{4}, H_{\theta}$ is explicitly invariant under uniform $S U(3)$ transformations on the spins [15], while for $\theta= \pm \frac{\pi}{2}, H_{\theta}$ is explicitly invariant under staggered conjugate rotations of the two sublattices 16]. These symmetries are hard to identify in the original spin representation but are evident in the $\mathrm{HC}$ bosonic representation.

The case where $\theta=\frac{\pi}{4}$ corresponds to the FM $S U(3)$ Heisenberg Hamiltonian, and therefore the GS is the state with maximum total $S U(3)$ spin $\mathcal{S}$. The OP associated with this broken symmetry is the total $S U(3)$ magnetization $\mathcal{S}^{\mu \nu}=\frac{1}{N_{s}} \sum_{\mathbf{j}} \mathcal{S}^{\mu \nu}(\mathbf{j})$ which has eight independent components. When $\left\langle\mathcal{S}^{\mu \nu}\right\rangle \neq 0$, the system orders, and the coexistence of a FM phase and a BE condensation becomes more evident: In the bosonic language both OPs correspond to different components of

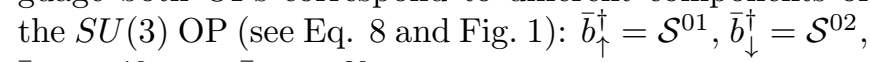
$\bar{b}_{\uparrow}=\mathcal{S}^{10}$, and $\bar{b}_{\downarrow}=\mathcal{S}^{20}$, are the components of the OPs for the $\uparrow$ and $\downarrow$ BE condensates, while $s^{z}=\frac{1}{2}\left(\mathcal{S}^{11}-\mathcal{S}^{22}\right)$, $s^{x}=\frac{1}{2}\left(\mathcal{S}^{12}+\mathcal{S}^{21}\right)$, and $s^{y}=\frac{1}{2 i}\left(\mathcal{S}^{12}-\mathcal{S}^{21}\right)$, are the components of the FM OPs for the bosons. The ability of $\left\langle\bar{n}_{\mathbf{j}}\right\rangle=\frac{2}{3}-\left\langle\mathcal{S}^{00}(\mathbf{j})\right\rangle$ to take any value during $S U(3)$ rotations of the GSs is another manifestation of the BE condensation.

We will now work to answer the question: What is the OP of the model in the original $S=1$ language (Eq. [1)? We start by writing the relation between the components of $\mathcal{S}^{\mu \nu}$ and the $S=1$ generators of $S U(2)$. From Eqs. 2 and 8 we have

$$
\begin{aligned}
S^{x} & =\frac{1}{\sqrt{2}}\left(\mathcal{S}^{01}+\mathcal{S}^{20}+\mathcal{S}^{02}+\mathcal{S}^{10}\right), \\
S^{y} & =\frac{1}{\sqrt{2} i}\left(\mathcal{S}^{01}+\mathcal{S}^{20}-\mathcal{S}^{02}-\mathcal{S}^{10}\right), \\
S^{z} & =\mathcal{S}^{11}-\mathcal{S}^{22}, \\
\left(S^{x}\right)^{2}-\frac{2}{3} & =\frac{1}{2}\left(\mathcal{S}^{12}+\mathcal{S}^{21}+\mathcal{S}^{00}\right),\left(S^{z}\right)^{2}-\frac{2}{3}=-\mathcal{S}^{00}, \\
\left\{S^{x}, S^{y}\right\} & =i\left(\mathcal{S}^{21}-\mathcal{S}^{12}\right), \\
\left\{S^{x}, S^{z}\right\} & =\frac{1}{\sqrt{2}}\left(\mathcal{S}^{01}-\mathcal{S}^{20}-\mathcal{S}^{02}+\mathcal{S}^{10}\right), \\
\left\{S^{y}, S^{z}\right\} & =\frac{1}{\sqrt{2} i}\left(\mathcal{S}^{01}-\mathcal{S}^{20}+\mathcal{S}^{02}-\mathcal{S}^{10}\right) .
\end{aligned}
$$

The first three operators correspond to the $S=1$ FM OP, while the second five are the components of the spinnematic OP (components of the bilinear symmetric traceless tensor). The traceless condition of $\mathcal{S}^{\mu \nu}$ implies that $\left(S^{y}\right)^{2}-\frac{2}{3}=-\left(\left(S^{z}\right)^{2}-\frac{2}{3}\right)-\left(\left(S^{x}\right)^{2}-\frac{2}{3}\right)$. This classification is independent of the parity and time-reversal properties of $\Lambda^{a b}(t)=S^{a}(t) S^{b}(0)(a, b=x, y, z)$ 17]. We see that by rotating the $S U(3)$ FM OP, we find a FM or a spin-nematic GS for the $S=1$ Hamiltonian. Moreover, we also see that the existence of a spin-nematic quantum phase is directly related to the $\mathrm{BE}$ condensation of the two-flavored HC bosons. Concomitantly, we have rigorously established the existence of a spin-nematic phase, an issue that had been outstanding in the literature [18].

Unveiling Order and Establishing Complexity. With the phases of $H_{\theta}$ established at the point $\theta=\frac{\pi}{4}$ we will now detail the nature of the phases near it as revealed by adding a symmetry breaking field to the model. A vast array of rich behavior is discovered, and we now highlight some of the more interesting results.

Coupling our two-flavored bosonic system $H_{1}$ to a magnetic field $\mathbf{B}$ through a Zeeman term removes the degenerancy of the FM GS associated to the $S U(2)$ symmetry. The resulting GS is a BE condensate polarized along the magnetic field direction (chosen to be the longitudinal $z$ direction). In fact, in the limit $|\mathbf{B}| \rightarrow \infty$, the 
resulting Hamiltonian is $H_{t-V}=t \sum_{\langle\mathbf{i}, \mathbf{j}\rangle}\left(\bar{b}_{\mathbf{i} \uparrow}^{\dagger} \bar{b}_{\mathbf{j} \uparrow}+\bar{b}_{\mathbf{j} \uparrow}^{\dagger} \bar{b}_{\mathbf{i} \uparrow}\right)+$ $V \sum_{\langle\mathbf{i}, \mathbf{j}\rangle}\left(\bar{n}_{\mathbf{i} \uparrow}-\frac{1}{2}\right)\left(\bar{n}_{\mathbf{j} \uparrow}-\frac{1}{2}\right)$ and represents a gas of $\mathrm{HC}$ bosons with hopping $t=J$ and nearest-neighbor densitydensity interaction $V=2 J$.

By means of the Matsubara-Matsuda transformation [2], we can map $H_{t-V}$ onto the FM $S=\frac{1}{2}$ Heisenberg model which has an exact solution. Therefore, $\mathbf{B}$ removes the $S U(3)$ invariance of $H_{1}$ and leaves an $S U(2)$ invariance associated to the charge degrees of freedom. In this way we recover the well-known connection between a FM $S=\frac{1}{2}$ state in the transverse direction and a BE condensate of spinless HC bosons. Because of the $S U(2)$ invariance of the Heisenberg model, the FM states polarized along $z$ are also GSs, so if we infinitesimally increase the attractive interaction between bosons, the model phase segregates (Ising-like ferromagnetism).

For $V=0, H_{t-V}$ reduces to an $\mathrm{XY}$ model whose GS is again a BE condensate (i.e., has off-diagonal long range order 19]). Regardless of the dimension of the system, the GS of $H_{t-V}$ is a BE condensate for $-2|t| \leq V \leq 0$ and phase segregates when $V<-2|t|$. In fact, for a very small repulsive interaction the GS is still a BE condensate [19]. In a similar fashion $H_{1}$ is at the point separating the $\mathrm{BE}$ condensate from phase segregation, i.e., if we make the two-body nearest-neighbor interaction infinitesimally more attractive, there is phase segregation.

A possible realization of the single-flavor bosonic system $H_{t-V}$ is the attractive $U$ Hubbard model in the large $|U|$ limit [20] with $t=-2 \tau^{2} /|U|$ and $V_{1}=4 \tau^{2} /|U|(\tau$ is the hopping integral of the original Hubbard model). It is clear that if we add a nearest-neighbor attractive interaction $v=-2 \tau^{2} /|U|$ to the original Hubbard model, we can exactly compute the superconducting GS and quasiparticle excitations in any spatial dimension and for any concentration of particles since $V=V_{1}+4 v=2 t$. In the case where $v=-\tau^{2} /|U|$ and $V=0$, the Hamiltonian is an XY model whose GS displays long-range order [19].

Conclusions. We presented an algebraic framework aimed at uncovering the order behind the potential multiplicity of complex phases in interacting quantum systems [21]. In this framework the local Hilbert space of dimension $N$ admits a convenient hierarchical representation in terms of the generators of an $S U(N)$ group [5]. We illustrated this for $N=3$ by first presenting the exact solution of a non-trivial model of interacting quantum spins. A key point is that from this hierarchical group $(S U(3)$ in the present case), one can identify the embedded subgroups (an invariant $S U(2)$ for spin and an $S U(2)$ for charge and two others connecting them), thereby establishing a hierarchical classification of order parameters.

Through the spin model, we rigorously demonstrated the possible coexistence of ferromagnetism and BE condensation of $s=\frac{1}{2}$ hard-core bosons. This condensate was shown to be related to a quantum spin-nematic ordering in the original spin model. These results may shed light on the physics associated with the recent surprising experimental observation of the coexistence of superconductivity and ferromagnetism in $\mathrm{UGe}_{2}$ [22].

We close with the observation that a fundamental relation between magnetism and $\mathrm{BE}$ condensation has emerged as a consequence of our spin-particle transformations: If a solution of a particular localized quantum spin $S$ model displays long-range order in the transverse direction $\left(\lim _{\left|\mathbf{r}_{\mathbf{i}}-\mathbf{r}_{\mathbf{j}}\right| \rightarrow \infty}\left\langle S_{\mathbf{i}}^{a} S_{\mathbf{j}}^{a}\right\rangle \neq 0\right.$, with $\left.a=x, y\right)$, then the corresponding itinerant bosonic model displays longrange phase coherence (i.e., a BE condensation). This result is general and independent of the sign of the exchange couplings in the spin model.

We thank D. Pines for a useful discussion. This work was sponsored by the US DOE under contract W-7405ENG-36. Part of it was performed while G. O. was a visitor at the Institute for Theoretical Physics (UCSB) which is supported by the grant No. PHY99-07949.

[1] P. Jordan and E. Wigner, Z. Phys. 47, 631 (1928).

[2] T. Matsubara and H. Matsuda, Prog. Theor. Phys. 16, 569 (1956).

[3] C.D. Batista and G. Ortiz, Phys. Rev. Lett. 86, 1082 (2001); Condensed Matter Theories, Vol. 16 (in press).

[4] These dictionaries (isomorphisms) translate spin language $S$ into itinerant quantum particle language with "effective $\operatorname{spin"} s=S-\frac{1}{2}$.

[5] C.D. Batista and G. Ortiz, cond-mat/xxxxxx.

[6] For example, for the standard Jordan-Wigner transformation any local physical operator is a linear combination of $\mathbb{1}, S_{\mathbf{i}}^{x}, S_{\mathbf{i}}^{y}$, and $S_{\mathbf{i}}^{z}$.

[7] A. Süto, J. Phys. A: Math. Gen. 26, 4689 (1993).

[8] N. Papanicolaou, Nucl. Phys. B 305, 367 (1988).

[9] In terms of standard boson operators $b_{\mathbf{j} \sigma}^{\dagger}, \mathrm{HC}$ in each fla$\operatorname{vor}\left(\left(b_{\mathbf{j} \sigma}^{\dagger}\right)^{2}=0,\left[b_{\mathbf{i} \sigma}, b_{\mathbf{j} \sigma^{\prime}}\right]=0\right.$, and $\left[b_{\mathbf{i} \sigma}, b_{\mathbf{j} \sigma^{\prime}}^{\dagger}\right]=\delta_{\mathbf{i j}} \delta_{\sigma \sigma^{\prime}}(1-$ $\left.\left.2 n_{\mathbf{i} \sigma}\right)\right)$ with the single occupancy constraint $n_{\mathbf{i} \uparrow} n_{\mathbf{i} \downarrow}=0$, these HC boson operators are: $\bar{b}_{\mathbf{j} \sigma}^{\dagger}=b_{\mathbf{j} \sigma}^{\dagger}\left(1-n_{\mathbf{j} \bar{\sigma}}\right)$. They satisfy: $\left[\bar{b}_{\mathbf{i} \sigma}, \bar{b}_{\mathbf{j} \sigma^{\prime}}\right]=0,\left[\bar{b}_{\mathbf{i} \sigma}, \bar{b}_{\mathbf{j} \sigma^{\prime}}^{\dagger}\right]=\delta_{\mathbf{i j}}\left(1-2 \bar{n}_{\mathbf{i} \sigma}-\bar{n}_{\mathbf{i} \bar{\sigma}}\right)$ (if $\left.\sigma=\sigma^{\prime}\right)$, or $-\delta_{\mathbf{i} \mathbf{j}} \bar{b}_{\mathbf{i} \sigma^{\prime}}^{\dagger} \bar{b}_{\mathbf{i} \sigma}\left(\right.$ if $\left.\sigma \neq \sigma^{\prime}\right)$.

[10] Any linear combination of states with different $\mathcal{N}$ and $S_{z}$ is a possible GS.

[11] To compute expectation values the following commutator is useful: $\left[\bar{b}_{\mathbf{j} \sigma},\left(\tilde{b}_{\mathbf{0} \sigma}^{\dagger}\right)^{\mathcal{N}_{\sigma}}\right]=\frac{\mathcal{N}_{\sigma}}{\sqrt{N_{s}}}\left(\tilde{b}_{\mathbf{0} \sigma}^{\dagger}\right)^{\mathcal{N}_{\sigma}-1}\left(1-\bar{n}_{\mathbf{j}}-\right.$ $\left.\bar{n}_{\mathbf{j} \sigma}\right)-\frac{\mathcal{N}_{\sigma}\left(\mathcal{N}_{\sigma}-1\right)}{N_{s}}\left(\tilde{b}_{\mathbf{0} \sigma}^{\dagger}\right)^{\mathcal{N}_{\sigma}-2} \bar{b}_{\mathbf{j} \sigma}^{\dagger}$.

[12] In the Cartan treatment of Lie algebras [13, the Cartan subalgebra of $S U(3)$ has rank 2 and our generators are $\mathcal{S}^{11}=\mathrm{H}_{1}$ and $\mathcal{S}^{22}=\mathrm{H}_{2}$ while the remaining off-diagonal elements of the matrix Eq. 8 represent the step operators $\mathrm{E}_{i}(i=1,2, \cdots, 6)$. These eight generators form the Cartan-Weyl basis of the Lie algebra $s u(3)$.

[13] J.F. Cornwell, Group Theory in Physics (Academic Press, San Diego, 1997).

[14] The conjugate fundamental representation of $S U(3)$ cor- 
responds to perform the transformation $\bar{b}_{\mathbf{j} \sigma}^{\dagger} \rightarrow-\bar{b}_{\mathbf{j} \bar{\sigma}}^{\dagger}$ $(\sigma=\uparrow, \downarrow)$ and conjugation [5]. The usual Jordan-Wigner transformation is an automorphism in $S U(3)$ that connects two $S U(2)$ conjugate subalgebras, one associated to the spin and another to the charge degrees of freedom. In this way, the Jordan-Wigner trasformation is like an internal symmetry.

[15] B. Sutherland, Phys. Rev. B 12, 3795 (1975).

[16] I. Affleck, Nucl. Phys. B 265, 409 (1986).

[17] A.V. Balatsky and E. Abrahams, Phys. Rev. Lett. 74, 1004 (1995).

[18] A.V. Chubukov, Phys. Rev. B 43, 3337 (1991); G. Fáth and J. Sólyom, Phys. Rev. B 51, 3620 (1995).

[19] T. Kennedy, E.H. Lieb, and B.S. Shastry, Phys. Rev. Lett. 61, 2582 (1988).

[20] P. Nozières and S. Schmitt-Rink, J. Low Temp. Phys. 59, 195 (1985).

[21] There exists an algebraic formulation of how to identify OPs that applies to mean-field Hamiltonians only. See, for example, Dynamical Groups and Spectrum Generating Algebras, edited by A. Bohm, Y. Ne'eman, and A.O. Barut (World Scientific, Singapore, 1988).

[22] S.S. Saxena et al., Nature 406, 587 (2000). 